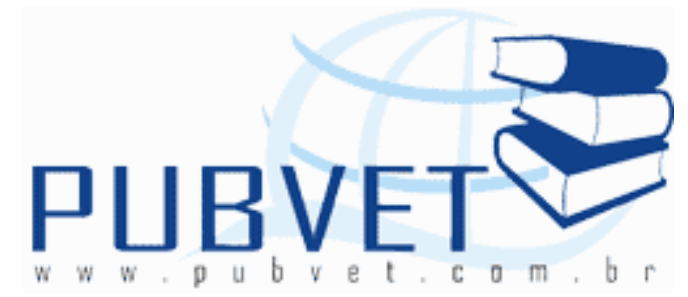

PUBVET, Publicações em Medicina Veterinária e Zootecnia.

\title{
Reutilização de pessários vaginais após autoclavagem sobre taxas reprodutivas em ovinos
}

Gustavo Desire Antunes Gastal*, Carine Dahl Corcini, Raquel Schiavon Schiavon, Virgilio Balduíno Scheid Filho, Rafael da Rosa Ulguim, Karina Lemos Goularte, Thomaz Lucia Jr.

ReproPEL, Faculdade de Veterinária, Campus Universitário s/n - Caixa Postal 354. CEP 96010-900 - Universidade Federal de Pelotas, Pelotas/RS. Brazil *Corresponding author: gastalgustavo@gmail.com

\section{Resumo}

O objetivo deste estudo foi avaliar o efeito da reutilização de pessários vaginais impregnados com progesterona $\left(\mathrm{CIDR}-\mathrm{G}^{\circledR}\right)$ após a sua esterilização por autoclave em diferentes protocolos de sincronização de estro em ovelhas. Dois experimentos foram conduzidos utilizando os seguintes protocolos: T1 foi sincronizado com pessários vaginais, cloprostenol e eCG , T2 utilizou o mesmo protocolo do T1 sem aplicação de eCG e T3 recebeu apenas cloprostenol. No segundo experimento todos os pessários vaginais foram esterilizados por autoclave, após a utilização no primeiro experimento. No primeiro experimento, a taxa de deteç̧ão de estro diferiu entre T2 e T3 $(81,0 \%$ e $65,0 \%$, respectivamente) $(P>0,05)$, porém esses grupos não diferiram do T1 $(67,9 \%)(P>0,05)$. No experimento 2 as taxas de detecção de estro do T1 $(89,0 \%)$ não diferiu de $\mathrm{T} 2(89,0 \%)(\mathrm{P}>0,05)$, sendo estas maiores que a do 
T3 (64,3\%). A taxa de prenhez de T1 $(68,0 \%)$ foi maior que T3 $(38,9 \%)$ $(P>0,05)$, mas a taxa observada em T2 (56\%) não diferiu das anteriores $(P>0,05)$. O uso de eCG não afetou a expressão de estro. O uso de pessários vaginais esterilizados por autoclave foi associado com melhor expressão de estro nos animais tratados, além de reduzir possíveis transmissões de doenças.

Palavras-chave: Estro. Sincronização. Autoclave. CIDR-G ${ }^{\circledR}$. Reutilização.

\section{Reuse of vaginal devices after autoclaving on rates parameters in sheep}

\section{Abstract}

The objective of this study was to evaluate the effect of used progesteronereleasing vaginal devices (CIDR-G ${ }^{\circledR}$, Pfizer) after autoclave sterilization in different estrous synchronization protocols in ewes. Two experiments were conducted using the following treatments: T1, synchronized with vaginal devices along with cloprostenol and eCG; T2, the same treatment of T1 without eCG; and T3, with cloprostenol only. In the second experiment, all vaginal devices were the same previously used in the first experiment, after autoclave sterilization. In the first experiment, the estrous detection rate differed between $\mathrm{T} 2$ and $\mathrm{T} 3(81.0 \%$ and $65.0 \%$, respectively) $(\mathrm{P}<0.05 \%)$, although both rates did not differ from T1 (67.9\%). In the second experiment, the estrous detection rate did not differ between T1 and T2 (89.3 and 89.3\%, respectively) ( $P>0.05)$, but both rates were greater than that for T3 $(64.3 \%)$ $(\mathrm{P}<0.05)$. The pregnancy rates for $\mathrm{T} 1 \quad(68.0 \%)$ was greater than for T3 (38.9\%) $(P<0.05)$, but the rate observed for $\mathrm{T} 2(56 \%)$ did not differ from the other rates $(P>0.05)$. The use of eCG did not affect estrous expression. Autoclave sterilization of vaginal devices was related to improved estrous expression in the treated animals and avoids the risk of transmission diseases.

Keywords: Estrous. Synchronization. Autoclave. CIDR-G ${ }^{\circledR}$. Reutilization. 


\section{INTRODUÇÃO}

A eficiência reprodutiva na espécie ovina pode ser otimizada, a partir da associação de programas de inseminação artificial (IA) e sincronização de estro, permitindo a redução no efeito da estacionalidade reprodutiva e a intensificação do manejo (MORAES et al., 2002). A sincronização do estro pode ser realizada mediante o fornecimento de progesterona ou seus análogos sintéticos por um período de cinco a sete dias (MENCHACA \& RUBIANES, 2001). O método de fornecimento mais usual é via implantes intravaginais (pessários) (MORAES et al., 2002; DIXON et al., 2006). Especialmente em épocas que não coincidem com a estação reprodutiva, utiliza-se a gonadotrofina coriônica eqüina (eCG) para incrementar o crescimento folicular (LEYVA et al., 1998; VIÑOLES et al., 2001). Já dentro da estação e em períodos em que a fêmeas estão ciclando, é necessários associar análogos da prostaglandina $\mathrm{F} 2 \mathrm{a}$ para eliminação da fonte de progesterona endógena (DIXON et al., 2006).

Porém os tratamentos hormonais apresentam resultados variáveis no desempenho reprodutivo do rebanho e ainda são considerados onerosos quando a relação custo/benefício é avaliada. Em conseqüência disso, alguns pesquisadores avaliaram a viabilidade da reutilização de dispositivos e implantes hormonais para sincronização do estro. Pinna et al. (2008) avaliaram a reutilização do CIDR por uma única vez em ovinos e obtiveram bons resultados de fertilidade, porém não foi utilizado nenhum processo de esterilização entre as utilizações do implante. Segundo Zuluaga e Williams (2008) a esterilização de CIDR antes da reutilização disponibiliza maiores concentrações de P4 imediatamente após a inserção em bovinos e reduz ao máximo o risco de transmissão da doença.

Este trabalho avaliou a eficiência da reutilização de pessários vaginais impregnados com progesterona (CIDR-G ${ }^{\circledR}$ ) após a sua esterilização por autoclave em diferentes protocolos de sincronização de estro em ovelhas, buscando identificar a efetividade da sincronização de estro e a taxa de prenhez obtida após inseminação artificial. 


\section{MATERIAL E MÉTODOS}

Animais e manejo

Foram utilizadas 174 ovelhas da raça Ideal (1-6 anos de idade) alojadas em uma propriedade em Capão do leão (RS) (janeiro de 2006) e 168 ovelhas da raça Texel (1-6 anos de idade) alojadas em uma propriedade localizada em Piratini (RS) (janeiro de 2007). Em ambas as propriedades os animais estavam sob regime extensivo de criação, com pastoreio em campo nativo e fornecimento de água e sal mineral ad libitum.

As fêmeas foram isoladas do contato com machos por 90 dias e após este período submetidas à indução da atividade sexual através do efeito macho durante 21 dias, utilizando-se machos adultos, castrados e androgenizados pela aplicação de $250 \mathrm{mg}$ de testosterona. As fêmeas que responderam a indução da ciclicidade, foram selecionadas mediante o uso de rufiões (1:15), preparados com tinta marcadora no peito. Os animais selecionados foram aleatoriamente alocados nos três tratamentos de sincronização.

Protocolos de sincronização

Foram realizados dois experimentos que constaram dos mesmos protocolos de sincronização, diferindo apenas em relação ao período de realização e rebanho. Foram compostos grupos de sincronização destinados a sincronização com implantes impregnados com progesterona e outro sem o dispositivo de progesterona.

Os implantes intra-vaginais foram mantidos in loco por seis dias, sendo que no momento de sua remoção os animais receberam a aplicação de um agente luteolítico $(0,133 \mathrm{mg}$ de D-Cloprostenol sódico) e uma aplicação de hormônio gonadotrófico eCG (250 UI de Novormon), compondo o tratamento1 (T1). Outro grupo de animais (T2) foram sincronizados com o mesmo protocolo do T1, porém sem aplicação de eCG. As demais ovelhas selecionadas receberam apenas uma dose de agente luteolítico (T3), simultaneamente a aplicação nos animais que foram submetidos ao tratamento com progesterona.

No experimento 1 os animais pertencentes aos grupos T1 e T2 receberam dispositivos intra-vaginais de progesterona novos. Já no experimento 2 , os 
dispositivos usados foram os mesmos do experimento 1 , sendo no entanto submetidos a autoclavagem $\left(121^{\circ} \mathrm{C}\right.$ a $724 \mathrm{mmHg}$ por 20 minutos) e armazenamento asséptico até sua reutilização.

$O$ retorno ao estro foi observado por dois ciclos estrais subseqüentes à sincronização (35 dias), com o uso de rufiões, utilizando tinta de cor diferente da utilizada anteriormente para o uso do efeito macho.

Nos dois experimentos, a detecção de estro foi realizada com uso de rufiões, por cinco dias, a partir da retirada dos pessários, duas vezes ao dia, com intervalo de $12 \mathrm{~h}$ entre as observações. Os rufiões eram pintados, todos os dias, na região medial do esterno, com tinta marcadora. No experimento 1 , foi medida apenas a taxa de expressão de estro. No segundo experimento, a IA ocorreu 12 horas após a detecção do estro, com sêmen fresco. A gestação foi determinada através de ultrassonografia em modo B (Anser Vet 485, Pie Medical ${ }^{\circledR}$ ), com transdutor convexo de $5,0 \mathrm{MHz}$, pela via transcutânea, 45 dias após a última IA.

As taxas de expressão de estro (em ambos os experimentos) e de prenhez (no segundo experimento) foram comparadas entre os tratamentos através do teste de qui-quadrado, usando o software Statistix ${ }^{\circledR}$ (2003).

\section{RESULTADOS}

No primeiro experimento, a taxa de expressão de estro do T2 foi superior à observada para o T3 $(P<0,05)$ (Tabela 1$)$. Porém, a taxa de expressão de estro observada no T1 não diferiu daquelas obtidas nos demais tratamentos ( $P$ $>0,05)$.

No segundo experimento, a taxa de expressão de estro não diferiu entre T1 e T2, porém foi inferior no T3 $(P<0,05)$, conforme mostra a Tabela 1. A Tabela 1 mostra que a taxa de prenhez observada para o $\mathrm{T} 1$ foi superior à observada para o T3, mas a taxa observada para o T2 não diferiu das demais $(P>0,05)$. 
Tabela 1. Taxas de expressão de estro (\%) e de prenhez (\%) nos tratamentos $(1,2$ e 3 , respectivamente) realizados nos experimentos 1 e 2 . (Rates of estrous expression (\%) and pregnancy (\%) in the treatments $(1,2$ and 3 , respectively) made in the experiments 1 and 2.)

\begin{tabular}{llll}
\hline Tratamento & $\mathrm{n}$ & Estro $(\mathrm{n})$ & Prenhez $(\mathrm{n})$ \\
\hline Experimento 1 & & & \\
PGF2a+P4+eCG & 56 & $67,9(38)^{\mathrm{ab}}$ & - \\
PGF2a+P4 & 58 & $81,0(47)^{\mathrm{a}}$ & - \\
PGF2a & 60 & $65,0(39)^{\mathrm{b}}$ & - \\
Experimento 2 & & & \\
PGF2a+P4+eCG & 56 & $89,0(50)^{\mathrm{a}}$ & $68,0(34)^{\mathrm{a}}$ \\
PGF2a +P4 & 56 & $89,0(50)^{\mathrm{a}}$ & $56,0(28)^{\mathrm{ab}}$ \\
PGF2a & 56 & $64,3(36)^{\mathrm{b}}$ & $38,9(14)^{\mathrm{b}}$ \\
\hline
\end{tabular}

PGF2a - 0,133mg de cloprostenol sódico, um análogo de prostaglandina F2a; P4 - Progesterona natural (0,33g CIDR-G ${ }^{\circledR}$, Pfizer); eCG - 250 UI Gonadotrofina Coriônica eqüina.

Experimento 1 - utilizado pessário impregnado de progesterona novo.

Experimento 2 - utilizado pessário impregnado de progesterona oriundo do experimento1.

$\mathrm{a}, \mathrm{b}$ Letras diferentes dentro de cada grupo e coluna diferem significativamente $\mathrm{P}$ $<0,05$.

\section{DISCUSSÃO}

As taxas de expressão de estro observadas neste trabalho foram maiores quando se utilizou o pessário autoclavado ( $\mathrm{T} 1$ e $\mathrm{T} 2$ ) que provavelmente pode se atribuído ao processo de esterilização á vapor que aumenta a taxa de eluição da progesterona (ZULUAGA \& WILLIAMS, 2008). Este efeito pode ser atribuído a modificações na estrutura ou na disposição da P4 no implante 
devido ao processo de esterilização a vapor. (ZULUAGA \& WILLIAMS, 2008). Além de que a reutilização dos CIDR tem potencial de transmitir microrganismos patógenos que podem disseminar doenças no rebanho. Todavia, não há aparentemente nenhum relatório da transmissão de doenças relativo à reutilização da inserção de CIDR.

O uso de eCG não influenciou a taxa de expressão de estro em comparação com o tratamento incluindo somente um análogo de um luteolítico. Além de que o uso de eCG em uma concentração de 250 UI no momento entre a retirada dos pessários foi associado com redução no intervalo até a expressão do estro. Resultados semelhantes foram observados por URIBE-VELÁSQUEZ et. al. (2002), que utilizaram o dobro da dose deste experiemento (500 UI de eCG), observando um aumento significativo das concentrações plasmáticas de progesterona e estradiol no inicio da fase luteal e um ciclo estral subseqüente com intervalo menor que o observado em fêmeas não tratadas.

Associação entre progestágenos e análogos de PGF2a, com ou sem o uso de eCG, proporcionou maior expressão de estro, em comparação com o protocolo convencional, apenas com o uso de luteolítico. Isso se justifica pelo fato de que, análogos de PGF2a somente teriam efeito em fêmeas que apresentam corpo lúteo responsivo, o que não ocorre normalmente no início da estação reprodutiva, quando nem todas as fêmeas que estão ciclando, ou quando aquelas que estão ciclando não se encontram no estágio de diestro (URIBE-VELÁSQUEZ et al, 2002). Ainda que a associação de luteolítico ao progestágeno tenha beneficiado a expressão de estro, o uso de eCG não apresentou benefício. As taxas de prenhez observadas no segundo experimento, com uso de progestágeno associado a um análogo de PGF2a e eCG indicam que houve um benefício na taxa de prenhez, em relação à taxa observada no grupo controle (LUTHER et al, 2007), mas o uso de eCG não trouxe benefício, se comparado ao T2, apesar de seu potencial efeito sobre o crescimento folicular (MCNATTY et al., 1982). 
GASTAL, G.D.A. et al. Reutilização de pessários vaginais após autoclavagem sobre taxas reprodutivas em ovinos. PUBVET, Londrina, V. 7, N. 13, Ed. 236, Art. 1554, Julho, 2013.

\section{CONCLUSÃO}

A esterilização a vapor contribui para a reutilização do CIDR obtendo uma melhor resposta na sincronização do estro em ovinos além de reduzir ao máximo o risco de transmissão de doenças.

\section{REFERÊNCIAS}

DIXON, A.B. et al. Reproductive performance of ewes after 5-days treatment with intravaginal inserts containing progesterone in combination with injection of Prostaglandin $F 2 \infty$. Reprod Dom Anim, v.41, p.142-148, 2006. http://www3.interscience.wiley.com/cgibin/fulltext/118611934/PDFSTART.

LEYVA, $V$. et al. Follicular activity and ovulation regulated by exogenous progestagen and PMSG in anestrous ewes. Theriogenology, v.50, p.377-93, 1998. Disponível em: <http://dx.doi.org/10.1016/S0093-691X(98)00147-2>. Acesso em 08 dec. 2009. doi:10.1016/S0093-691X(98)00147-2.

LUTHER, J.S. et al. The effect of GnRH, eCG and progestin type on estrous synchronization following laparoscopic AI in ewes. Small Rumin Res, v.72, p.227-231, 2007. Disponível em: <http://dx.doi.org/10.1016/j.smallrumres.2006.10.015>. Acesso em 15 dec. 2009. doi:10.1016/j.smallrumres.2006.10.015.

MACNATTY, G.B. et al. Preovulatory follicular development in sheep treated with PMSG and/or prostaglandin. J Reprod Fert, v.65, p.111-123, 1982. Disponível em: <http://www.reproduction-online.org/cgi/reprint/65/1/111>. Acesso em 12 dec. 2009.

MENCHACA, A.; RUBIANES, E. Effect of high progesterone concentrations during the early luteal phase on the length of the ovulatory cycle of goats. Anim Reprod Sci, v.68, p.69-76, 2001. Disponível em: <http://dx.doi.org/10.1016/S0378-4320(01)00139-7>. Acesso em 12 dec. 2009. doi:10.1016/S0378-4320(01)00139-7.

MORAES, J.C.F. et al. Controle do estro e da ovulação em bovinos e ovinos. In: GONÇALVES, P.B.D. et al. Biotécnicas aplicadas à reprodução animal. Ed. Varela, São Paulo, 2002, p.25-55.

PINNA, A.E.; BRANDÃO, F.Z.; CAVALCANTI, A.S. Fertilidade de ovlehas cíclicas submetidas à sincronização do estro utilizando implantes intravaginais (CIDR) novos ou reutilizados. Acta Scientiae Veterinariae 36, suplemento 2. 2008.

STATISTIX®. Statistix $® 8$ Analytical Software. Tallahassee. FL. 2003. 396 p.

URIBE-VELÁSQUEZ, L. F. et al. Respostas endócrinas e ovarianas associadas com o folículo dominante da primeira onda folicular em ovelhas sincronizadas com CIDR ou PGF2 $\infty$. Rev Bras Zootec, v.31, n.2, p.944-953, 2002. Disponível em: $<$ http://www.scielo.br/scielo.php?script=sci abstract\&pid=S151635982002000400018\&lng=pt $\underline{\&} \mathrm{nrm}=\mathrm{iso} \& \mathrm{t} \mid \mathrm{ng}=\mathrm{pt}>$. Acesso em $05 \mathrm{dec}$. 2009. doi: 10.1590/S1516-35982002000400018.

VIÑOLES, C. et al. Effect of long-term and short-term progestagen treatment on follicular development and pregnancy rate in cyclic ewes. Theriogenology, v.55, p.993-1004, 2001. Disponível em: <http://dx.doi.org/10.1016/S0093-691X(01)00460-5>. Acesso em $11 \mathrm{dec}$. 2009. doi:10.1016/S0093-691X(01)00460-5.

ZULUAGA, J.F.; WILLIAMS, G.L. Hidh-pressure steam sterilization of previously used CIDR inserts enhances the magnitude of the acute increase in circulating progesterone after insertion in cows. Animal Reproduction Science, 107 p.30-35, 2008. 